\title{
Criminologie
}

\section{Femmes et emprisonnement : le marquage du corps et l'automutilation}

\section{Sylvie Frigon}

Volume 34, numéro 2, automne 2001

Comportements suicidaires et délinquance

URI : https://id.erudit.org/iderudit/027504ar

DOI : https://doi.org/10.7202/027504ar

Aller au sommaire du numéro

\section{Éditeur(s)}

Les Presses de l'Université de Montréal

ISSN

0316-0041 (imprimé)

1492-1367 (numérique)

Découvrir la revue

Citer cet article

Frigon, S. (2001). Femmes et emprisonnement : le marquage du corps et l'automutilation. Criminologie, 34(2), 31-56. https://doi.org/10.7202/027504ar
Résumé de l'article

À l'aide d'entrevues avec des femmes incarcérées dans un établissement correctionnel provincial, l'auteure nous invite à réfléchir sur les effets de l'incarcération sur le corps des femmes et plus particulièrement sur la signification de l'automutilation. Cet article situe l'automutilation chez les femmes incarcérées dans une perspective plus globale du marquage du corps en prison et comme un cas de figure de la trajectoire iden- titaire trouble de ces femmes. Dans cette perspective, le « corps " est conçu comme un site central de la manifestation du pouvoir pénal. Cette discussion se divise en quatre parties. Une première partie présente un profil des femmes incarcérées au Canada. Une deuxième retrace l'itinéraire théorique, méthodologique et épistémologique, emprunté dans cette démarche de recherche. Une troisième présente les résultats de la recherche sur ce corps enfermé autour de deux axes conceptuels : le corps comme site de contrôle et le corps comme site de résistance. Une quatrième et dernière partie tente de proposer des pistes pour penser l'automutilation dans une perspective plus large d'analyse.
Ce document est protégé par la loi sur le droit d'auteur. L'utilisation des services d’Érudit (y compris la reproduction) est assujettie à sa politique d'utilisation que vous pouvez consulter en ligne.

https://apropos.erudit.org/fr/usagers/politique-dutilisation/ 


\title{
Femmes et emprisonnement : le marquage du corps et l'automutilation
}

\author{
Sylvie Frigon \\ Professeure \\ Déportement de criminologie \\ Université d'Ottawa \\ sfrigon@uottawa.co
}

RÉsumé - À l'aide d'entrevues avec des femmes incarcérées dans urr établissement correctionnel provincial, l'auteure nous invite à réfléchir sur les effets de l'incarcération sur le corps des femmes et plus particulièrement sur la signification de l'automutilation. Cet article situe l'automutilation chez les femmes incarcérées dans une perspective plus globale du marquage du corps en prison et comme un cas de figure de la trajectoire identitaire trouble de ces fermmes. Dans cette perspective, le « corps » est conçu comme un site central de la manifestation du pouvoir pénal. Cette discussion se divise en quatre parties. Une première partie présente un profil des femmes incarcérées au Canada. Une deuxième retrace l'itinéraire théorique, méthodologique et épistémologique, emprunté dans cette démarche de recherche. Une troisième présente les résultats de la recherche sur ce corps enfermé autour de deux axes conceptuels : le corps comme site de contrôle et le corps comme site de résistance. Une quatrième et dernière partie tente de proposer des pistes pour penser l'automutilation dans une perspective plus large d'analyse.

SUMmaRY - In the light of interviews conducted with provincially-sentenced women, the author invites the readers to examine the effects of incarceration on women's bodies and most particularly, the significance of self-mutilation. This article locates selfmutilation within a wider social perspective of the marking of the body and as an example of the troubled identity trajectory of these women. Moreover, the body is taken as a site of the manifestation of power. This discussion comprises four parts. The first part is a presentation of the profile of incarcerated women in Canada. The second one traces the theoretical, methodological and epistemological journey taken in this research project. The third one presents the research results on the topic of the imprisoned feminine body which is conceptually organised as the body as a site of control and the body as a site of resistance. The fourth and final part proposes ways of understanding selfmutilation within a wider social perspective. 
La prison agit de telle sorte qu'elle vous vide progressivement, y compris l'intérieur de vous-même. C'est la logique de l'enfermement d'aller jusqu'au squelette (Dr Gonin cité dans Ginsberg, 1992 : 129).

[...] la prison, c'est de la frustration et de la colère si intenses que de m'entailler les artères du bras ne fait que soulager un peu de cette souffrance (Témoignage d'une femme incarcérée à la Prison des femmes, cité dans Service correctionnel du Canada, $1990: 6$ ).

Il n'est pas étonnant que plusieurs d'entre nous se tranchent la gorge, se lacèrent le corps, se pendent. Il n'est pas étonnant que nous ayons besoin d'inscrire notre douleur sur notre corps, car notre vie est remplie d'une incroyable douleur et d'expériences traumatisantes - douleur psychique, douleur physique, douleur morale (Mme Cree, 1994: 47 - Notre traduction).

La prison marque et façonne le corps. Des femmes en prison marquent, mutilent leur corps. D'autres résistent. Nous allons tenter d'examiner comment le corps porte les traces des logiques et des structures pénitentiaires. Nous allons tenter de saisir les enjeux de cette emprise en donnant la parole aux femmes incarcérées. Le corps comme médium, comme intermédiaire par lequel passe la parole des femmes nous permettra de saisir les profondes significations de son insertion charnelle socio-pénale.

En prenant le corps comme pivot d'analyse et l'automutilation comme cas de figure, nous examinerons comment le corps des femmes devient transparent, pénétrable, assujetti, mais comment aussi il résiste à l'enfermement carcéral. Cet article situe l'automutilation chez les femmes incarcérées dans une perspective plus globale du marquage du corps en prison, comme un cas de figure de la trajectoire identitaire trouble des femmes et prend le « corps » comme site central de la manifestation du pouvoir pénal.

Afin de mieux comprendre cette problématique, nous allons explorer la voix des femmes incarcérées, dans le but de mieux saisir l'automutilation et les pratiques de marquage du corps adoptées par ces dernières comme stratégies de " résistance ». Notre discussion sera divisée en quatre parties. Une première partie présente quelques statistiques sur l'ampleur du phénomène de l'incarcération des femmes au Québec et au Canada. Une deuxième retrace notre itinéraire théorique, méthodologique et épistémologique. Une troisième présente les résultats de notre recherche sur ce corps enfermé organisé autour de deux axes conceptuels : le corps comme site de contrôle et le corps comme site de résistance. Une quatrième et dernière partie tente de proposer des pistes pour penser l'automutilation dans une perspective plus large d'analyse. 


\section{Des femmes incarcérées au Canada : quelques statistiques}

Au Canada, depuis les années 1970, le recours à l'incarcération pour les femmes justiciables a augmenté. En effet, au milieu des années 1970, les femmes ne représentaient que $2 \%$ de la population carcérale fédérale. En 1975, il y avait 174 femmes purgeant une peine fédérale en prison. En 1989, le nombre est passé à 273, puis à 322 en septembre 1995 (Shaw, 1991). En 1995, 305 femmes purgeaient des peines fédérales, contre 13234 hommes $^{1}$. Jusqu'en 1996, la Prison des femmes de Kingston était le seul pénitencier pour femmes au Canada, comparativement à plus de 40 établissements pout hommes. On y comptait habituellement environ 150 femmes (population francophone variant entre $20 \%$ et $40 \%$ ) et quelque 150 autres étaient incarcérées dans des établissements provinciaux à la suite d'accords d'échange conclus avec certaines provinces, comme le Québec depuis 1973. En 1998, le chiffre est passé à 360 femmes (Hannah-Moffat et Shaw, 2000).

Le 5 octobre 1996, les femmes représentaient $5 \%$ de tous les détenus inscrits au registre dans des établissements correctionnels canadiens fédéraux et provinciaux ( 1807 femmes pour 37541 détenus) (Trevethan, 1999). À titre d'exemple, à la Maison Tanguay à Montréal, on comptait entre avril et décembre 1996 (lorsque l'établissement détenait encore la population fédérale) 2,031 admissions et de ce nombre il y avait $90(4,4 \%)$ sentences d'emprisonnement discontinu (les fins de semaine), 896 ( $44,1 \%$ ) prévenues (en attente de procès), 888 $(43,7 \%)$ condamnées à des peines de moins de 6 mois, dont 545 $(61,4 \%)$ avaient moins de 30 jours de détention à purger, $100(4,9 \%)$ plus de 6 mois et moins de deux ans et $25(1,2 \%)$ plus de deux ans ${ }^{2}$. En 1998 , on comptait 14000 personnes devant purger une sentence fédérale, dont 360 femmes (2,5\% de la population fédérale).

Tout au long des années 1990, les femmes incarcérées représentai 7 de ta population carcérale provinciale (sentences de moins de deux ans) et $4 \%$ de la population carcérale fédérale (sentences de deux ans et plus). Il semble par ailleurs que l'on puisse noter à la fois une diminution des accusations criminelles et une augmentation du recours à l'incarcération.

1. Voir Statistiques Canada, $1990: 1$

2. Nous tenons à remercier Madame Aline White, conseillère spécialisée en milieu correctionnel à la Maison Tanguay, pour ces données. 
Il y a des points de convergence lorsque l'on examine les caractéristiques sociales des hommes et des femmes en prison, particulièrement en ce qui concerne la pauvreté, les niveaux élevés de chômage, les faibles niveaux de scolarisation, les difficultés psycho-sociologiques des familles, les antécédents de toxicomanie et les taux élevés de suicide et de dépression. Par ailleurs, comme nous le verrons, certaines caractéristiques sont uniques aux femmes.

Jusqu'au milieu des années 1990 approximativement, le Canada était doté d'un seul établissement carcéral fédéral complètement fonctionnel, ce qui posait un certain nombre de difficultés. Parmi celles-ci, on pense particulièrement à l'éloignement des femmes de leur famille, de leur milieu social d'origine et des programmes de réinsertion sociale locaux. Les femmes provenaient de toutes les provinces canadiennes ; les conséquences pour les détenues mères étaient très sérieuses et souvent néfastes pour elles et leurs enfants. Cela concerne beaucoup de femmes en détention, puisque les deux tiers des femmes purgeant une peine au fédéral sont mères et que $70 \%$ de celles-ci élèvent seules leurs enfants (Arbour, 1996). De plus, contrairement aux hommes, elles n'ont généralement pas de conjoint à l'extérieur qui s'occupe des enfants. La population carcérale féminine est plus jeune et plus susceptible d'avoir la charge légale et morale des enfants.

Une autre difficulté, en termes de gestion d'un unique établissement fédéral, concernait la classification. Toutes les femmes incarcérées étaient soumises au même type de contrôle, même si les besoins de sécurité étaient différents, contrairement à la situation qui existe pour les hommes. Ceci avait des conséquences aussi bien en termes de mobilité dans l'institution, d'accès aux différents programmes de travail, de formation et d'activités sociales que de possibilités de visites familiales.

Par ailleurs, c'est le nombre non négligeable de tentatives de suicide et de suicides observés entre 1977 et 1991 , à la Prison des femmes, qui a sonné l'alarme. Selon des sources non officielles, une douzaine de femmes, dont huit autochtones (elles représentent environ $25 \%$ des femmes au fédéral et sont donc largement surreprésentées), se seraient enlevé la vie à l'intérieur des murs ${ }^{3}$. Comme nous l'avons mentionné plus tôt, selon le rapport d'un groupe d'étude sur les femmes ayant subi une sentence au fédéral - La création de choix - plus de $80 \%$ des femmes incarcérées auraient été victimisées avant leur incarcération. La violence qu'elles

3. Voir Faith, $1994: 139$. 
ont subie, la plupart du temps aux mains d'bommes (par exemple, de la part du père, d'un frère, d'un beau-père ou d'un conjoint), ne devrait donc pas être passée sous silence, lorsque l'on parle d'intervention auprès des femmes.

En 1990, le rapport intitulé La création de choix recommandait la fermeture de la Prison des femmes de Kingston pour l'année 1994 (elle fut finalement fermée en 1996) et l'ouverture de six centres régionaux de détention au Canada. Les nouveaux centres sont situés à la réserve de Nekaneet, en Saskatchewan, pour les femmes autochtones (Pavillon de ressourcement), à Edmonton en Alberta, à Joliette au Québec, à Kitchener en Ontario et à Truro en Nouvelle-Écosse. Un centre pour femmes en Colombie-Britannique était déjà en opération depuis 1991 (Service Correctionnel du Canada, 1990 ; Service Correctionnel du Canada, 1995) ${ }^{4}$. Cette fermeture de la Prison des femmes avait d'ailleurs été préconisée depuis son ouverture en 1934. Plusieurs commissions d'enquête se sont succédées pour toutes en arriver à la même conclusion : la fermeture de la Prison des femmes ${ }^{5}$.

Malgré les changements de modalités d'incarcération et la nouvelle philosophie pénale en matière de services correctionnels pour femmes, plusieurs difficultés persistent (Martel, 1999 ; Hannah-Moffat et Shaw, 2000), tels, par exemple, le nombre élevé de tentatives de suicide et de suicides, l'(in)accès aux programmes, les relations avec les enfants, le recours à l'isolement cellulaire et aux unités à sécurité maximale. Des difficultés similaires se retrouvent du côté des femmes incarcérées dans des établissements provinciaux. Comme nous allons pouvoir le constater, les difficultés se vivent jusque dans le corps de la femme détenue.

\section{Itinéraire théorique, méthodologique et épistémologique}

Notre recherche vise à explorer comment l'investissement disciplinaire du corps des femmes s'articule, en théorie et en pratique, dans un univers disciplinaire particulier, à savoir la prison. La bande vidéo produite sur les événements survenus à la Prison des femmes de Kingston, en avril 1994, montre graphiquement l'assujettissement des corps des femmes et la production de « corps dociles » (Arbour, 1996 ; Frigon, 1997, 1999a

4. Voir aussi Stableforth (1999), pour un bilan récent de la situation des services correctionnels pour femmes au Canada.

5. Dès 1938 , le rapport Archambault recommandait la fermeture de P4W. 
et 2000). Pour les fins de notre présentation, nous allons prendre l'automutilation comme cas de figure du marquage du corps.

\subsection{L'ancrage théorique}

À l'instar de Foucault (1975) et de bien des auteurs d'inspiration foucaldienne, nous sommes d'avis que tout un ensemble de procédures dans l'enfermement participe à l'opération d'assujettir les corps pour les rendre à la fois "dociles et utiles ». Dans ce sens, l'héritage foucaldien et la littérature post-disciplinaire (voir Garland, 1997, pour une revue de la littérature sur le sujet) sont précieux. Mais comment cette discipline se traduit-elle sur le corps féminin ? Là-dessus, la plupart des auteurs sont restés assez silencieux et font l'objet de critiques (Bartky, 1990 ; Bordo, 1993 ; Ramazanoglu, 1993 ; Grosz, 1994 ; Howe, 1994 ; Hannah-Moffat et Shaw, 2000).

En traçant des repères théoriques essentiels, nous nous proposons d'explorer ce que les femmes identifient comme pertinent pour elles dans cette " anatomie politique du corps ». Cette recherche s'ancre dans la quotidienneté (Smith, 1987 et 1990), dans l'expérience des femmes et non seulement dans l'histoire de l'assujettissement des corps. De plus, le but de la recherche est d'examiner les processus et mécanismes de contrôle et de résistance qui opèrent au niveau du corps des femmes dans un cadre institutionnel particulier, afin de mieux saisir les processus disciplinaires sociaux. Elle vise aussi à traiter de la question du corps et de l'emprisonnement, de la construction sociale et pénale de la féminité en mettant en relief la production de " corps dociles » en criminologie. Ici, les travaux de Carlen (1988), Hamelin (1989), Faith (1994) et Howe (1994), pour ne nommer que ceux-ci, sont éclairants à propos de l'emprisonnement des femmes.

La sociologie du corps est un domaine en plein essor et les études théoriques et empiriques sur le corps constituent un domaine de plus en plus important dans la recherche féministe (Rail, 1998 ; Frigon et Kérisit, 2000). Les domaines de la maternité (Quéniart, 1988) et des nouvelles technologies de reproduction (Vandelac 1986, 1993 ; Weir, 1996) sont aussi des exemples de domaines de recherche appliquée. Dans l'ensemble des textes, par ailleurs, l'accent est souvent mis sur le contrôle et l'oppression, sans que l'on comprenne comment ce contrôle s'opère au quotidien. Par conséquent, les stratégies de résistance, de négociation et de subversion que développent les femmes dans leurs pratiques corporelles sont ignorées. Dans notre recherche, les femmes ne 
sont pas perçues uniquement comme des victimes, mais aussi comme des actrices sociales à part entière dans les rapports de pouvoir. Ainsi, la réflexion proposée dans les pages qui vont suivre s'inscrit-elle dans la continuation de cette démarche.

La question du corps a tenu et tient toujours une place importante dans les savoirs et pratiques criminologiques sans être, par ailleurs, réellement explorée. Les liens entre le corps et le crime, le corps comme lieu d'explication de la déviance, marginalité et criminalité ont fait leur apparition bien avant le XIX ${ }^{e}$ siècle. Dès lors, comme le souligne Labadie (1995), une phrénologie du mal et une vraie cartographie du crime émergent. En effet, d'hier à aujourd'hui, les savoirs criminologiques et les pratiques pénales qui s'y rapportent s'appuient, en partie, sur une lecture, une connaissance des corps à divers niveaux, souvent pour différencier - marquer une différence - entre le délinquant et le non-délinquant, entre le conforme et le déviant, entre nous et eux, entre le bon et le méchant .

Qui plus est, nous avons vu qu'historiquement, le corps des " criminels" était tantôt surveillé, tantôt contrôlé, tantôt torturé et même décapité. Toutefois, au début du XIX ${ }^{\mathrm{e}}$ siècle, ce châtiment-spectacle est remplacé. Il y a donc transition de la macropolitique du spectacle à la microphysique d'une surveillance, comme le souligne Foucault (1975). La torture est ainsi remplacée par la peine de mort, dépourvue de tout ce théâtre de supplices antérieur (on passe par la guillotine, puis par la pendaison, la chaise électrique et des techniques plus sophistiquées comme les injections). L'emprisonnement devient une forme plus humanitaire de punition. Certes, il n'en reste pas moins que la gestion symbolique fondamentale du corps demeure dominante dans les pratiques pénales ${ }^{6}$. Cette nouvelle philosophie de punition requiert l'intervention de toute une batterie d'intervenants, incluant notamment surveillants, éducateurs, criminologues, conseillers spécialisés, psychologues et psychiatres, notamment.

Mais il ne faut pas s'y méprendre : l'enfermement concerne toujours le corps - privation de liberté, contrôle des mouvements, rationnement alimentaire, absence d'intimité, privation sexuelie, coups, cachots, fouilles à nu. Par le truchement du corps, on veut atteindre l'âme dans ce rituel pénal (Laplante, 1985, 1995). Pour expliquer cette transformation, Foucault parle d'une «technologie politique du corps» dans

6. Nous n'avons qu'à penser à l'introduction récente du bracelet électronique comme stratégie de surveillance des contrevenants. 
laquelle on vise la docilité, l'obéissance, la soumission, mais aussi la production. L'assujettissement est réalisé aussi bien par la force physique que par un système calculé et technique.

Le corps féminin, pour sa part, fut objet de fascination et de fouilles, mesures, observations et classement par Lombroso et d'autres qui nous livrent une typologie de la criminalité féminine. Pour Lombroso, par exemple, la femme criminelle est toutefois bien plus terrible que l'homme, parce qu'elle transgresse son rôle de femme, épouse et mère. Des caractères de dégénérescence sont attribués au corps de la femme criminelle, qui est scruté, fouillé et tient lieu d'explication de la criminalité et plus encore. En effet, ce qui finalement émerge des travaux de Lombroso semble être beaucoup moins la pathologie de la femme criminelle que la "dangerosité » potentielle des femmes dites «normales » (Horn, 1995 : 109). Ainsi, toutes les femmes pourraient devenir les cibles et les objets de régulation et de surveillance tant du regard scientifique que des pratiques sociales ${ }^{7}$. C'est ce que l'on peut appeler la fonction symbolique du corps déviant et criminel des femmes (Préjean, 1994). Or toutes les femmes deviennent « suspectes ». D'autres auteurs de l'époque prendront le relais.

En Angleterre, dans les années 1860, des tenants de la physiognomonie, tels Carpenter, Robinson et Mayhew, font des liens entre l'apparence physique et les tendances criminelles. Pour sa part, Carpenter parle des femmes comme d'une classe de personnes exclues, physiquement dégénérées et moralement corrompues. Ellis aussi suggéra que ces femmes étaient plus ataviques, plus poilues, plus masculines (Dobash $e t$ al., 1986). Ainsi, la masculinisation du corps des femmes criminelles est centrale dans l'analyse de ces auteurs et de cette période historique. Ellis lie la criminalité des femmes à leur biologie. Maudsley, pour sa part, fut un des premiers médecins britanniques à identifier les fonctions normales du corps des femmes comme une cause d'aliénation et de folie. Les menstruations, la grossesse, par exemple, pouvaient conduire, selon lui, à une condition pathologique. En effet, il conclut que les déviations sexuelles des femmes sont le produit « de l'irritation des ovaires ou de l'utérus - une maladie qui transforme la femme chaste et modeste par une rage incontrôlée d'appétit " (ibid. : 114 - Notre traduction). Selon Dobash et al.,

7. Voir Hutter et Williams (1981) et Schur (1984) sur ce sujet. 
[l']analyse des femmes fut particulièrement importante dans ces écrits, car la femme, dans son rôle de mère, était considérée comme la cause biologique et sociale de la dégénérescence, et les hypothèses patriarcales sur la vraie nature des femmes conduisaient à des tentatives pour identifier et classifier celles qui déviaient de cette norme (1986: 111 - Notre traduction).

Cette conception, voulant que les femmes criminelles soient ontologiquement différentes, corrompues physiquement et psychologiquement, aura un impact certain sur le traitement des femmes en prison ; elle renvoie aussi à une image de femmes justiciables plus difficiles, voire même irrécupérables (voir également Smart, 1976 ; Heidensohn, 1985).

Même si l'enfermement se présente comme une forme plus humanitaire de punition et que l'on assiste à un adoucissement des peines, dans un contexte de changement social, politique et idéologique, c'est toujours du corps dont il s'agit.

\subsection{Parcours méthodologiques et épistémologiques}

L'ancrage de cette recherche se trouve dans la perspective des femmes («feminist standpoint » ou féminisme de l'expérience) (Harding 1986, 1987, 1991 ; Smith 1987). L'approche qualitative de type biographique (récits de vie) a été favorisée dans la construction de notre objet, car l'enquêtée est au centre de l'analyse. De plus, nous avons choisi l'entretien non directif, car il nous offrait une plus grande ouverture et flexibilité et parce qu'il permettait l'exploration de sujets complexes. Cette technique part du postulat que l'enquêtée est la mieux placée pour parler du sujet, car elle le vit, l'enquêteur jouant le rôle de facilitateur. À l'aide d'une consigne large et d'une grille thématique complémentaire, nous avons exploré un certain nombre de thèmes et sous-thèmes, car une série d'hypothèses et de questions nous préoccupait. Au chapitre de l'échantillonnage, nous avons tenté de diversifier la provenance des interviewées. Nous avons réalisé des entretiens avec 25 femmes incarcérées dans une prison provinciale, la Maison Tanguay à Montréal ( 2 ans moins un jour), de profils différents (jeunes et moins jeunes, séjours courts et longs, multiples incarcérations, première incarcération, différents délits, ayant ou non purgé des peines d'incarcération fédérales, au début et à la fin de la peine, mères, célibataires, hétérosexuelles et homosexuelles, de classes défavorisées et favorisées et de différents groupes ethniques), sans toutefois demander de préciser en détail toutes ces données factuelles. Les entretiens enregistrés se sont déroulés durant 
l'été 1998. La durée des entretiens varie entre une heure et deux heures. Nous avons tenu à explorer les expériences des femmes dans une prison provinciale palliant ainsi l'absence relative de recherches par rapport à celles de plus en plus nombreuses sur la situation des femmes ayant reçu une sentence fédérale ( 2 ans et plus). Par ailleurs, les données recueillies ont des points de convergence avec les expériences des femmes dans des institutions fédérales; de plus, certaines femmes de notre échantillon avaient déjà purgé une sentence fédérale.

Afin de préserver l'anonymat, des noms fictifs ont été attribués et les détails personnels ont été éliminés ${ }^{8}$. Dans les prochaines pages, nous proposons une réflexion théorique autour de la notion de "corps", nourrie par la perspective des femmes, dans le but de réfléchir sur les effets de l'incarcération dans le quotidien par le truchement de leur corps et sur la signification de l'automutilation. Il importe de préciser que la recherche porte sur le thème du corps en général et que l'automutilation est l'un des sous-thèmes de recherche.

\section{Le corps enfermé comme site de contrôle et comme site de résistance}

Les récits des femmes nous montrent comment le corps est un site central de la manifestation du pouvoir et comment, par le marquage, la transformation et la mutilation des corps, s'installe et se perpétue le pouvoir de punir. Dans cette perspective, nous allons mettre en relief plusieurs dispositifs de contrôle du corps. Comment l'enfermement travaille-t-il sur le corps ? Comment ce corps est-il investi ? Comment est-il contrôlé, fouillé ? Comment ce corps est-il à la fois un site de contrôle et un site de résistance? Le corps peut-il servir aussi d'outil de survie et même de résistance ? Il semble que diverses stratégies,

8. Nous tenons à remercier toutes les femrnes qui ont accepté de participer à la recherche. Nous tenons également à remercier les assistantes de recherche Brigitte Lavigne et Marie-Josée Frenette, qui ont contribué aux différentes étapes de la recherche (voir en particulier Lavigne, 1999). Pour l'analyse, nous nous sommes inspirée du texte de Paillé (1994) portant sur l'analyse par théorisation ancrée. Cette approche est une adaptation de la grounded theory proposée en 1967 par Glaser et Strauss. Selon Paillé, théoriser a [c]'est dégager le sens d'un événement, c'est lier dans un schéma explicatif divers éléments d'une situation, c'est renouveler la compréhension d'un phénomène en le mettant différemment en lumière " (1994: 149). Et, de poursuivre Paillé, " [q]uelque soit le niveau de théorisation atteint, le résultat devra être ancré (grounded) solidement dans les données empiriques recueillies " (ibid: 150). 
particulièrement liées aux corps, sont utilisées par les femmes pour se réapproprier un sens d'identité.

Dans le but de situer la parole des femmes sur la question du positionnement corporel dans une institution carcérale, nous avons organisé notre analyse sur deux axes : le corps enfermé comme site de contrôle et le corps comme site de résistance. Dans le premier axe, c'est-à-dire le corps comme site de contrôle, les thèmes sont réunis autour de quatre catégories : 1 - le corps marqué ; 2 - le corps aliéné ; 3 - le corps malade ; 4- le corps victime. Dans le deuxième axe, le corps comme site de résistance est analysé à un moment clé, pendant l'automutilation.

\subsection{Le corps comme site de contrôle}

1. Le corps marqué

Le marquage du corps peut se réaliser de diverses manières, assurant ainsi son intégration sociale : scarifications, tatouages, circoncision, mutilations sexuelles, reconstructions chirurgicales de parties du corps, perforation du corps et de certaines de ses parties (lèvres, oreilles, langue, parties génitales) et ornementation du corps. Ces inscriptions corporelles remplissent ainsi diverses fonctions selon l'espace social et culturel dans lequel elles s'inscrivent.

Les rites d'entrée en prison sont de véritables « cérémonies de dégradation " au sens de Garfinkel (1956). Pour Nicole, une femme incarcérée, c'est l'humiliation :

Là, un moment donné, ben la surveillante a dit bon là, passe à douche [...] j't'allée m'laver, mais y fallait s'déshabiller devant eux autres. Ça, ça m'a écœurée pas possible [...] le fait d'être obligée de s'déshabiller devant une surveillante, surtout tu connais pas cette personne-là [...] c'est presque une humiliation, un manque de respect envers la personne, la détenue (citée dans Hamelin, 1989 : 128-129).

Pour Angel et Ève, interviewées lors de notre recherche, la fouille :

[...] c'est une atteinte à ton intimité, te faire pencher, pis te faire écartiller, je déteste ça [...] "Lève les bras, les dessous des pieds, penche toé, écartille toé ". Ça m'enrage, je déteste ça, c'est humiliant, c'est humiliant (Angel).

Pis « lève les seins, écartes les fesses, pis lève tes cheveux, pis ouvre ta bouche, lève ta langue, lève tes bras pis tes pieds » (Ève).

Les femmes parlent beaucoup de l'humiliation vécue lors des fouilles à nu et des fouilles vaginales-rectales, ce qui explique l'importance que 
nous lui avons accordée. Les fouilles peuvent s'effectuer à l'entrée en prison comme tout au long de l'incarcération après des absences temporaires et des libérations de jour, par exemple. Nikita, également interviewée pour notre recherche, raconte : «[...] me mettre à nu, c'est déjà humiliant surtout quand moi j'suis plus grasse, disons que j'ai trouvé ça très dur, très dur ». En effet, pour Laurence et pour bien d'autres, la fouille

[...] c'était juste pour écœurer parce que en réalité le vaginal-rectal c'est une grosse farce, la fille peut n'avoir et l'infirmière qui le fait, c'est juste pour dire qu'elle rentre son doigt et même encore si elle le rentre, des fois c'est assez loin qu'elle peut même pas y toucher, faque c'est tout simplement pour humilier l'individu (dans Hamelin, 1989 : 130).

Le témoignage suivant est aussi fort éloquent sur ce thème :

Je suis passée à la fouille, j'étais hyper gênée, tu peux pas t'imaginer ! La gardienne elle me dit : "déshabillez-vous », t'es gênée, tu vois? Alors tu sais je lui ai dit : « j'ai mes règles, j'ai du sang », elle m'a même (pas) laissée finir, elle m'a dit "ouh la j'ai l'habitude, c'est pas...", tu vois ? J'aurais eu une merde sur la tête..., rien à taper. [...] Je me sentais sale. Je me sentais plus rien. [...] Tout, tu perds tout. J'avais plus de seins, j'avais plus de fesses, j'avais plus rien. [...] J'avais plus de corps" (témoignage d'une ex-détenue dans Welzer-Lang et al,, $1997: 22$ ).

Dans notre étude, cette dimension est aussi mise en évidence. En effet, pour Isabelle, la fouille pendant les règles est très gênante, humiliante :

La semaine passée, j'avais mes règles et je passe à la cour. Tsé tu te déshabilles, t'enlèves ta serviette sanitaire, tu la mets dans un papier, $y$ t'en donne une autre, y te regarde là tout le temps que tu fais ça, faut qu'elle voit s'il y a vraiment du sang dans la serviette sanitaire [...]. Ah, j'étais gênée (Isabelle).

Au Québec, le Protecteur du citoyen estimait également, dans son rapport de 1985 (p.204), que la fouille à nu, justifiée ou non, est toujours humiliante. Les rites d'entrée, les fouilles à nu et les fouilles vaginales-rectales participent donc de ce processus de marquage et de mortification et entraînent une perte de statut et d'identité. Ainsi, les femmes ne se perçoivent plus comme des femmes, mais comme des criminelles, ainsi qu'en témoigne Marie Gagnon, une ex-détenue de la Maison Tanguay à Montréal :

Je me regarde dans le miroir. Je ne suis plus la même personne. J'ai maintenant les yeux durs, les prunelles sombres; mes joues sont plus 
rondes, ce qui n'empêche pas deux gros sillons de les relier au nez. Je suis plus lourde, mais j'ai l'air amaigrie [...]. Peu à peu, je me transforme en ermite. J'ai les couleurs de Tanguay peintes sur ma peau. Je suis pâle. Mes cheveux sont secs et ternes [...] (1997: 112).

Ces dispositifs servent à la mise en forme de la punition qui produit la docilité, l'obéissance et la soumission (Foucault, 1975) et, comme le dit si bien Ève, " [...] c'est pas correct, c'est hypocrite, c'est pour montrer qui ont le pouvoir ". Avec le corps marqué, nous avons une autre épaisseur de cette expérience qui produit ce corps aliéné.

\section{Le corps atiéné}

"Qu'il me traite de même comme si j'étais un lépreux, ça ça m'écoeure, ça me pue au nez " (Ève).

Comme nous l'avons vu, les fouilles participent d'une logique de mortification. Mais bien d'autres mécanismes produisent le corps aliéné, voire transparent, des femmes incarcérées. Par exemple, à FleuryMérogis, " [a]vant 1974, les miroirs n'étaient pas autorisés ! Une femme pouvait passer un an, cinq ans, dix ans sans voir son visage " (Ginsberg, 1992 : 153). Le docteur Gonin explique que l'incarcération produit " un enfermement sensoriel et une désertification du goût, de l'odorat, du regard [...]" (ibidem : 129). Tous les sens sont perturbés. Pour Gayle Horii, une ex-détenue de la Prison des femmes de Kingston, la désertification du goût se manifeste ainsi :

Le parfum des fleurs, de l'encens, du thé au jasmin, la senteur de la peau de votre amoureux - - tout est remplacé par l'odeur de désinfectant, de moisissure, d'eau nauséabonde et de nourriture gâtée, de papier poussiéreux et de savon d'hôpital qui pénètre dans vos narines. On ne peut faire autrement que d'être disloquée (Horii, 1994: 7- Notre traduction).

Les fermmes se sentent épiées, surveillées. Marie Gagnon le raconte de cette façon :

J'écris dans le secteur. Il ressemble à une grande salle d'observation. Des vitres nous séparent du contrôle d'où les screws peuvent à loisir nous examiner. Je retourne en cellule où, du moins, je ne me sens pas dans le monde d'Orwell " (op.cit: 118).

Le corps est aussi aliéné parce qu'il devient asexué : les relations intimes entre les femmes sont peu tolérées. À titre d'exemple, à FleuryMérogis, jusqu'en 1984, on procédait à la ségrégation des lesbiennes en les plaçant dans le « quartier de garçonnes » (Ginsberg, 1992 : 156). Le 
manque d'intimité est aussi relaté dans nos entrevues. Ce manque d'intimité provoque le sentiment de ne pas exister, d'être transparente et sans importance comme en témoigne Ève :

J'étais assise sur la toilette et un gardien, un homme, est rentré dans ma cellule. Il y avait un Playboy sur ma table. Moi, j'étais assise pis il a fait comme si de rien n'était, il est rentré dans ma cellule, il a pris le Playboy de la fille qui était avec moé dans ma cellule. Il commence à le feuilleter pis il commence à me parler. Pis, j'suis assise sur la toilette, tsé. J'ai les culottes à terre pis j'sais pas quoi faire (rire). Là t'sais, j'sais pas, j'me lève-tu pour lever mes culottes ou quoi ? Fait que j'suis restée assise sur la toilette là, je me suis penchée tsé, $j$ 'ai attendu qu'il sorte.

Bien d'autres mécanismes créent l'aliénation du corps. Mentionnons, pour terminer cette section, le corps-mère. Les femmes enceintes sont perçues comme étant ontologiquement différentes, voire déviantes (Finateri, 1999), les femmes venant d'accoucher ou même les femmes ayant des enfants (Fournier, 2000), comme inadéquates. En effet,

The body of the imprisoned woman however, is not only inferior in relation to that of the "normal body" but also in relation to the conceptualization of the "good" woman. A positivistic, scientific view of the body denotes " criminal " bodies as ontologically inferior to "law" abiding bodies. Under such an ideologically position, pregnant imprisoned women possess bodies that reflect a qualitative and quantitative multiplication of levels of inferiority by virtue of their criminal "abnormality " or "atavism " and "feminine inferiority" (Finateri, $1999:$ 138).

Dans ce contexte, les mères subissent de profonds bouleversements par l'absence de l'enfant ; ces bouleversements se vivent jusqu'à la moelle de leurs os et de leur identité (voir à ce sujet Fournier, 2000).

\section{Le corps malade}

Même avant l'incarcération, le corps de ces femmes est mal en point en raison souvent de leur style de vie. Elles connaissent des problèmes de santé, souvent chroniques. Ainsi, des troubles de sommeil et d'alimentation, des problèmes dentaires, des infections gynécologiques et même de la séroposivité, liée parfois à la prostitution et aux toxicomanies et très souvent à la pauvreté et à la marginalisation, font partie du portrait clinique de ces femmes. Ces maux s'amplifient ou se développent pendant que se vit l'incarcération. Attardons-nous maintenant au corps pendant celle-ci. 
Durant l'incarcération, le corps parle. Divers troubles psychosomatiques apparaissent. Les mots/maux du corps reliés au stress (procès, enfants, conjoint, argent...) se manifestent. Par exemple, le stress lié aux conditions de détention engendre à son tour différents maux. En 1986, une équipe française de spécialistes est chargée d'une enquête sur "les pathologies somatiques et les conditions de vie en détention " où 867 hommes et 29 femmes sont interrogés. Parmi les pathologies signalées, on note la sensation de vertige, la désorientation, la difficulté à situer les événements récents, les maux de tête, les dérèglements du système digestif, des douleurs rénales et musculaires, des troubles de la sensorialité incluant la diminution du goût, une baisse de la vue et des troubles de l'audition 9 . Les troubles d'audition sont attribuables, en partie, aux bruits et aux rythmes de la vie carcérale. Selon Ginsgerg, " [...] les pas des surveillants, les chariots qui grincent, les portes qui s'ouvrent et se ferment, les clés qui tintent, l'oeilleton qui claque, les cris des détenus en crise ou emmenés au mitard [...] amplifiés par la forte résonance de l'espace carcéral " (ibidem : 128) font partie de la vie quotidienne carcérale.

Pour les femmes qui partagent une cellule dans laquelle on trouve des toilettes, les problèmes de constipation sont très sérieux, mais personne ne parle de ces banalités parce que cela est presque obscène ${ }^{10}$. Le témoignage de Paola dans notre recherche est éloquent sur ce point :

On attend aussi le soir pour aller aux toilettes, tu sais jamais quand quelqu'un va arriver. T'es jamais tranquille. Fait que ça nous stresse, ça affecte notre côté santé, aussi. J'veux dire pour aller à la selle là, on attend le soir. Y'a fait sa ronde, toutes les portes sont barrées, tout

9. "Au bout d'un an de détention, $79 \%$ des détenus se sentent, en moyenne, en mauvaise et très mauvaise santé, alors qu'ils étaient $54 \%$ à s'estimer en bonne santé et $31 \%$ en moyenne santé à leur arrivée. Lors de la mise sous écrou, une sensation de vertige envahit très souvent les personnes emprisonnées et la moitié d'entre elles, totalement désorientées, ont de la difficulté à situer les événements récents. Les troubles qui apparaissent au fil des mois sont des maux de tête (de 44 à $56 \%$ ), des dérèglements du système digestif $(40 \%)$, des douleurs rénales et musculaires ( $40 \%$ ), une impression de maigrir (30\%) et des douleurs vagues dans la poitrine. Des troubles de la sensorialité se manifestent après quelques mois de prison : $51 \%$ des détenus éprouvent une diminution du goût (nourtiture médiocre et fade oblige). Une baisse de la vue est signalée par 31 à $49 \%$ des prisonniers (chiffre variant selon le nombre de mois de prison que l'on a derrière soi). [...] 31 à $39 \%$ des détenus constatent une perte importante de leur odorat. Les troubles de l'audition semblent moins nombreux mais progressent au cours de la détention, passant de 15 à $28 \%$ [...] " (ibidem: 127-128).

10. Toujours, selon Ginsberg : "Les femmes sont constipées! Pas la petite rétention intestinale banale et brève, non, la constipation qui dure, persiste, fait gonfler le ventre et le rend dur comme une pierre, provoque des gaz et des bruits de " tuyauterie " invraisemblables [...] " (ibidem: 123). "Le manque d'intimité rend les femmes constipées» (ibidem: 124). 
l'monde est couché, on va à la toilette tranquille. Ça joue sur le système, tu t'habitues à te retenir tout le temps. Le soir c'est peut-être pas prêt à sortir mais c'est ton seul temps [...] (Paola).

Chez ces femmes, la perte d'intimité, la perte de contrôle sur leur corps et sur leur santé contribuent à produire un corps malade. En effet, selon le rapport intitulé $L a$ création de choix, les femmes affirment avoir perdu tout contrôle sur leur corps et leur santé (Service correctionnel du Canada, $1990: 53-54)^{11}$. La santé mentale est aussi fragilisée pendant l'enfermement. Dans la recherche menée par Martel (1999), des femmes racontent leur sentiment de perte de contrôle et l'impression de devenir folles pendant l'isolement cellulaire.

I couldn't sleep anymore, I was totally slept right out. And, I didn't eat. And I was trying to exercise. And, I was just like, fuck it, I'm going crazy [...] They treat you like shit in there, and it's like you know, it just gives you, like, this helpless, hopeless feeling... and the next thing you know it's, like, go into a state of depression (Agnes : 13 dans Martel, $1999: 68$ ).

L'expérience d'Agnes évoque l'idée que la dépression peut aussi servir de refuge ${ }^{12}$.

\section{Le corps victime}

Les femmes tolèrent mal l'enfermement. Les traumatismes qu'elles ont déjà vécus sont amplifiés par l'incarcération, car - nous l'avons déjà souligné - beaucoup de ces femmes ont été victimisées à un moment ou l'autre de leur vie (abus sexuels, inceste, violence conjugale). Pour Lyne, interviewée dans notre recherche, la prison lui rappelle la violence qu'elle a subie aux mains de son mari pendant vingt ans :

Quand je suis dehors, c'est pas pire. J'pense pas à ce que mon mari m'a faite. Mais quand je suis icitte, toute me revient (Lyne).

Pour les femmes autochtones, la situation est encore plus dramatique (Service correctionnel du Canada, 1990), comme en fait foi le témoi-

11. La séroposivité et le sida sont aussi au cour des problèmes de santé vécus par les femmes.

12. Selon une recherche menée auprès de 22 femmes usagères des ressources alternatives en santé mentale au Québec, Rodriguez del Barrio (2000) constate que la folie, et plus particulièrement la dépression, peuvent se vivre comme une souffrance, comme un corps envahi et en perte de contrôle, mais que la folie peut aussi se vivre comme refuge et/ou espace à soi (Rodriguez del Barrio, $2000: 183-186$ ). 
gnage d'une femme autochtone, cité en début d'article et que nous reprenons ici :

Il n'est pas étonnant que plusieurs d'entre nous se tranchent la gorge, se lacèrent le corps, se pendent. Il n'est pas étonnant que nous ayons besoin d'inscrire notre douleur sur notre corps, car notre vie est remplie d'une incroyable douleur et d'expériences traumatisantes - douleur psychique, douleur physique, douleur morale (Mme Cree, 1994: 47 - Notre traduction).

Ainsi, pour beaucoup de femmes, le système carcéral reproduit cette violence (Service correctionnel du Canada, 1990 ; Arbour, 1996). Telle est l'inéluctable continuité. En effet, les femmes racontent qu'elles sont revictimisées à nouveau pendant l'incarcération et plus particulièrement pendant l'isolement cellulaire (Martel, 1999).

Certaines femmes dans notre recherche expliquent que la détention est une autre forme de violence. Janice raconte que le système est violent et qu'il ne favorise pas la réinsertion sociale :

Déjà dans mon enfance, c'était pas beau... Là ben, avec le système qui est comme ça, disons que ça aide pas beaucoup pour la réhabilitation... ça fait peur de sortir après (Janice).

Ève ira même jusqu’à suggérer que le corps de la femme en général fait l'objet d'abus de différentes formes et dans diverses situations :

Le corps de la femme est abusé dehors, dans la rue, en prison, partout. J'étais danseuse, j'étais prostituée, pis j'l'sais (Ève).

Avec Comack (1996), nous pensons que les processus de victimisation et de criminalisation sont étroitement liés. Cette dimension est fort peu étudiée et mérite une attention particulière ${ }^{13}$.

Dans cette perspective, l'automutilation peut se manifester souvent comme des appels à l'aide et comme un signe de détresse. De plus, il est important de souligner que des femmes s'automutilaient alors qu'elles étaient en liberté et qu'elles reproduisent ce comportement à l'intérieur des murs (Daigle et al.,1999). Cette dimension mériterait une attention particulière.

Nous aurions pu discuter plus longuement du geste automutilatoire dans cette section, mais nous avons opté pour une analyse de cette

13. La violence exercée par les femmes en prison est aussi une problématique fort complexe, mais très peu explorée au Québec et au Canada (voir Croteau, 2000 pour un travail exploratoire sur la question). 
problématique sous l'angle de la résistance, car les femmes ont mis beaucoup l'accent sur cet aspect, comme nous le verrons dans la prochaine section.

\subsection{Le corps comme site de résistance}

Si les corps sont traversés et infiltrés par des savoirs, par des significations et par des pouvoirs, ils peuvent également, dans certaines circonstances, devenir le lieu de luttes et de résistances et faire euxmêmes leurs inscriptions sur les pratiques sociales. À la passivité du corps porteur d'inscriptions, il faut opposer l'activité d'un corps désirant et inscrivant qui, bien que marqué par la loi, laisse à son tour ses propres inscriptions sur le corps des autres, sur lui-même et sur la loi (Grosz, 1992: 56).

Le corps en prison sert également d'outil de survie et même d'outil de résistance. Diverses stratégies, particulièrement liées aux corps, sont utilisées par les femmes pour se réapproprier un sens d'identité. Le maquillage, l'habillement, les tatouages, les grèves de la faim et les relations intimes constituent de telles stratégies. Pour les besoins de notre exposé, nous nous attarderons ici à un moment clé où le corps peut devenir un site de résistance : l'automutilation.

\section{L'automutilation}

L'acteur en quête de sens et de valeur à donner à son existence interroge le signifiant ultime, le signifiant maitre : la mort, pour savoir (sans qu'il le sache toujours) [...] si vivre a encore une signification. Seule en effet la mort sollicitée symboliquement, à la manière d'un oracle, peut dire la légitimité d'exister [...] (Le Breton, $2000: 13$ ).

Les femmes incarcérées s'automutilent beaucoup ${ }^{14}$, plus que les hommes. Les hommes, en général, tournent leur violence vers les autres, tandis que les femmes la retournent contre elles-mêmes. Elles se punissent. En 1979, une étude ontarienne menée auprès de détenues d'un établissement provincial indiquait que $86 \%$ des femmes s'étaient déjà mutilées (Ross et McKay dans Faith, 1993 : 243). Dix ans plus tard, Jan Heney réalisait des entrevues auprès de 44 femmes subissant une sentence au fédéral : $59 \%$ s'étaient déjà mutilées; de ces quelque $60 \%$, $92 \%$ s'étaient entaillé les veines. D'autres se frappaient la tête, se brûlaient. Une étude récente menée par Daigle, Alarie et Lefebvre

14. La littérature est abondante à ce sujet (voir Kendall, 1993 et Faith, 1994 pour une recension). 
(1999) montre que le risque suicidaire moyen était plus élevé pour l'ensemble des femmes incarcérées au Québec (deux établissements provinciaux et un établissement fédéral) comparativement aux hommes incarcérés. Qui plus est, les femmes incarcérées dans des établissements provinciaux avaient les scores les plus élevés en termes de risque suicidaire.

Les femmes incarcérées marquent leur corps. Pour certaines, se couper, se blesser est une façon de s'approprier leur corps, d'exercer un certain contrôle sur celui-ci ${ }^{15}$. La femme peut décider quand elle se coupera, combien de fois, jusqu'où elle ira. Cela fait contraste avec toutes les autres occasions où elle a été victime, où elle n'avait pas de contrôle. Selon Heney (1990), l'automutilation est en effet une stratégie de survie. Comme le souligne une détenue dans le documentaire $\dot{A}$ double tour, "en prison, la pression est tellement forte qu'on finit par se déchirer le corps $\%$. C'est également une façon " pour faire face" et aussi le résultat de la violence subie pendant l'enfance (corps victime). Selon Pollack (1993), l'automutilation est une stratégie de dislocation du corps, de dissociation entre le corps et l'âme. Mme Cree, une femme autochtone, a en effet expliqué le sens que prenait l'automutilation dans la section corps victime. Ainsi, pour plusieurs femmes, l'automutilation provoque un sentiment de soulagement :

[...] la prison, c'est de la frustration et de la colère si intenses que de m'entailler les artères du bras ne fait que soulager un peu de cette souffrance (témoignage d'une femme incarcérée à la Prison des femmes, cité dans Service correctionnel du Canada, $1990: 6$ ).

Le témoignage de Sara dans notre recherche est aussi révélateur à ce sujet :

J'm'en souviens, on m'avait coupé mon code (congé) parce que mes papiers avaient été mal faits. Le système avait "fucké » [...]. J'ai travaillé tellement longtemps pour ça, pour en arriver là, on arrive on me dit ça avant Nöel, de même. Pis mon petit bonhomme m'a dit : "Ben j'pensais que t'étais mon cadeau pour Nöel ». Ben j'avais « pété une fuite" ", pis à place de la péter sur une autre, i'me suis en allée danis ma cellule, fallait que je fasse de quoi. Y'avait une rage en dedans de moi, fallait que ça sorte. Y'avait trop de mal fait que je me suis ouvert pour la première fois. Puis, ça faite "oouf ", ça faite comme un

15. Nous sommes consciente que la signification du geste peut être une pette de contrôle et/ou une réelle tentative de s'enlever la vie. Par ailleurs, nous avons voulu rendre compte d'autres significations qui sont aussi importantes pour les femmes. 
" release ", c'est comme si cela avait ôté une tension en dedans de moi [...]. Ça m'a même fait peur parce que c'est tellement trop un bon " release " (Sara).

Dans ce témoignage, plusieurs aspects ressortent : diriger sa rage vers soi au lieu d'autrui, prendre son corps comme site de soulagement et le soulagement provoqué par le geste mutilateur. Comme le dit Sara, « [j]'ai eu assez mal intérieurement que je revirais ça contre moi, fait que je mutilais mon corps pour débarquer la rage ". Ce témoignage illustre aussi le sentiment d'(im)puissance que les femmes ressentent. Souvent incapables d'avoir un certain contrôle sur les événements qui les entourent, les femmes se révoltent sur elles-mêmes, sur leur corps. Par exemple, lorsque Lyne s'est vu refuser la libération conditionnelle, elle s'est ouvert les veines. Elle raconte :

J'suis partie en tabarnak. J'étais dans ma cellule, mon rasoir était encore là, c'était la journée des rasoirs. J'ai pris mon rasoir, je me suis ouvert. J'ai fait comme ça (geste de s'ouvrir les veines). Je me suis coupée jusqu'ici, j'ai coupé ça, ça, pis ça (Lyne).

Pour Ève, le geste mutilateur est en réaction à une situation familiale difficile : au lieu de s'en prendre à sa mère ou de communiquer verbalement la douleur, elle s'en prend à elle-même.

Moé icitte j'ai fait ça avec une canette [...]. J'étais fâchée après ma mère parce que ma mère, elle m'aime pas [...] (Ève).

Dans ce processus d'autodestruction et de marquage, plusieurs femmes peuvent raconter leur vie par les marques de lacération : chaque cicatrice est le témoin de peines, de rages, d'impuissance, chaque coupure pouvant raconter des amours blessées, la douleur de l'absence des enfants, un Noël en prison ou un anniversaire manqué.

\section{Pour penser l'automutilation dans une perspective plus large d'analyse}

Comme nous pouvons le constater dans ces témoignages, plusieurs aspects émergent. Par exemple, les femmes ont besoin de diriger leur rage, leur peine vers elles au lieu d'autrui, d'avoir l'impression d'exercer un certain contrôle et de prendre leur corps comme un site de soulagement. Le soulagement provoqué par le geste mutilateur illustre aussi, souvent, le sentiment d'(im)puissance que les femmes ressentent. En effet, se sentant incapables d'avoir un certain contrôle sur les événe- 
ments qui les entourent, les femmes se révoltent contre elles-mêmes, contre leur corps. La structure et la logique carcérales amplifient le sentiment d'impuissance et les femmes sentent l'institution se resserrer autour d'elles comme un étau.

Dans ce contexte, nous proposons de penser l'automutilation dans une perspective plus large d'analyse, car les explications strictement psychologiques et les tests psychologiques, bien qu'essentiels, ne permettent de lever le voile que partiellement sur le phénomène de l'automutilation et celui du marquage du corps.

Premièrement, dans le geste mutilateur, mourir n'est souvent ni la finalité première ni même la finalité tout court ${ }^{16}$. Le geste mutilatoire n'est pas toujours une tentative de suicide dans le concret, mais un suicide symbolique - dans le but de s'effacer et de se rendre invisible. En effet, dans mon étude sur les représentations du corps et de l'identité des femmes incarcérées, le geste prend un autre sens en prison : la dislocation des repères. Dans un univers carcéral et totalitaire, l'individu finit par ne plus avoir de repères, de sens et doit donc par le fait même se fabriquer un certain sens. L'automutilation peut, dans certains cas, se vivre comme une quête de sens parce qu'il n'y a pas d'autre chose.

Selon Le Breton (2000), les conduites à risques (on peut analyser l'automutilation comme telle) peuvent également se comprendre comme un rapport aux limites - comme si jouer symboliquement avec la mort permettait de se reconstituer, de se sentir vivre. Ce "saut dans le vide", ce "moment de suspension ", pour reprendre des termes chers à Le Breton, permettent aux femmes de reprendre un certain contrôle ou, à tout le moins, d'avoir un sentiment de contrôle. Cette forme symbolique de reprise en main peut avoir aussi comme signification profonde de fabriquer du sacré dans un genre de rituel. Dans ce sens, le témoignage d'une des femmes interviewées dans le cadre de notre recherche est révélateur : elle raconte qu'avant de se mutiler, elle nettoie sa cellule, range ses choses et allume des chandelles. Le geste mutilateur est ritualisé.

Le geste mutilatoire peut être à la fois une façon de se rendre invisible et de se prendre en main dans le but d'exister. En apparence contradictoires, ces deux mouvements ou finalités s'interpellent parce que le geste mutilatoire prend toute sa signification dans celle même que lui confere l'actrice (acteur). De plus, il faut réfléchir sur le sentiment de soulage-

16. Le travail de LeBreton (2000) est utile pour commencer à penser ce phénomène. 
ment, aussi physique que psychique, que provoque le geste mutilateur, tel qu'il est exprimé chez certaines femmes. Ce sentiment de soulagement est conjugué à ce que l'on pourrait appeler une stratégie de dislocation du corps et de l'esprit - comme si l'univers fermé, contrôlé et opprimant dans lequel ces femmes vivent ne leur permettait l'évasion que de cette façon. Le corps et l'automutilation deviennent des refuges.

D'autres formes de marquage du corps, tel le "body piercing ", l'échange de seringue, le tatouage, les grèves de la faim, la musculation et le maquillage en prison mériteraient d'être également analysées pour en comprendre les sens et les significations profondes.

En épluchant les épaisseurs des dispositifs carcéraux, on lève le voile sur le corps comme site de contrôle et comme site de résistance. À travers les récits des femmes, on constate comment le corps des femmes est marqué, aliéné, et comment il devient transparent. Le processus de mortification associé au passage au pénal résulte en une perte d'identité. Cette recherche exploratoire montre comment la prison renvoie à une identité corporelle - un corps qui ne s'appartient plus. La production de l'identité à travers son propre regard et le regard de l'autre est donc une double production (identité personnelle et identité sociale).

Par ailleurs, le corps résiste à l'aliénation engendrée par la logique pénitentiaire. Les femmes ont plusieurs stratégies de survie quotidiennes et d'autres plus exceptionnelles (automutilation et tentatives de suicides). L'automutilation est un cas de figure intéressant, illustrant l'érosion du corps des femmes par le pouvoir de punir. Les quelques pistes de réflexion que nous avons proposées méritent d'être reprises afin d'en explorer les sens et enjeux, aussi bien personnels qu'institutionnels. Ainsi, comme nous pouvons le constater, le corps et, par extension, l'automutilation demeurent des lieux privilégiés de l'investissement pénal.

\section{Références}

Arbour, L. 1996. Commission d'enquête sur certains événements survenus à la Prison des femmes de Kingston, Canada : Ministère des Approvisionnements et Services.

BARTKY, S. 1990. Femininity and Domination, New York: Routledge.

BORDo, S. 1993. Unbearable Weight, Feminism, Western Culture, and the Body, Berkeley : University of California Press. 
Carlen, P. 1988. Women, Crime and Poverty, Milton Keynes : Open University Press.

CREE, MS. 1994. "Entrenched Social Catastrophe " Journal of Prisoners on Prisons 5 (2) : 45-48.

CROTEAU, C. 2000. Prison Violence in Canada : A female Inmate's Perspective, thèse de maîtrise inédite, Département de criminologie, Université d'Ottawa.

Daigle, M., Alarie, M. et Lefebvre, P. 1999. «La problématique suicidaire chez les femmes incarcérées " Forum 11 (3) : 41-45.

Debuyst, C., Digneffe, F., Labadie, J.-M. et PiRes, A. 1995. Histoire des savoirs sur le crime \& la peine, 1 . Des savoirs diffus à la notion de criminel-né, Les Presses de l'Université de Montréal, Les Presses de l'Université d'Ottawa, DeBoeck Université.

DOBASH, R.P., Dobash, R.E. et GutTERIDGe, S. 1986. The Imprisonment of Women, Oxford : Basil Blackwell.

FaITH, K. 1994. Unruly Women. The Politics of Confinement \& Resistance, Vancouver : Press Gang Publishers.

FINATERI, L. 1999. « The Paradox of Pregnancy in Prison : Resistance, Control, and the Body » Canadian Woman Studies/Les cabiers de la femme 19 ( 1 \& 2) : 136-144.

Foucault, M. 1975. Surveiller et punir. La naissance de la prison, Paris : Gallimard.

FOURNIER, J. 2000. L'expérience des mères justiciables : les perceptions du rôle de mère lors de l'incarcération et lors du retour en communauté, thèse de maîtrise inédite, Département de criminologie, Université d'Ottawa.

Frigon, S. 1997. "Sexe, mensonge et vidéo " Journal of Prisoners on Prisons $8(1 \& 2): 105-112$.

FrIGON, S. 1999. «Une radioscopie des événements survenus à la Prison des femmes : la construction d'un corps dangereux et d'un corps en danger " $C a-$ nadian Woman Studies/Les cabiers de la femme 19 (1 \& 1 2) : 154-160.

Frigon, S. 2000. "Corps, féminité et dangerosité : de la production de "corps dociles" en criminologie ", Pp.127-164 in Du corps des femmes : contrôle, surveillance et résistance, sous la direction de S. Frigon et M. Kérisit. Ottawa : Les Presses de l'Université d'Ottawa.

Frigon, S. et KÉRISIT, M. 2000. Du corps des femmes : contrôle, surveillance et résistance, Ottawa : Les Presses de l'Université d'Ottawa.

GAGNON, M. 1997. Bienvenue dans mon cauchemar, Montréal : VLB éditeurs.

GaRfinkel, H. 1956. "Conditions of Successful Degradation Ceremonies " The American Journal of Sociology LXI (5) : 420-424.

Garland, D. 1997. "'Governmentality' and the Problem of Crime : Foucault, Criminology, Sociology » Theoretical Criminology 1 (2) : 173-214.

GinsberG, G. 1992. Des prisons et des femmes, Paris : Éditions Ramsay.

Grosz, E. 1992. “Le corps et les connaissances. Le féminisme et la crise de la raison » Sociologies et sociétés XXIV (1) : 47-66. 
Grosz, E. 1994. Volatile Bodies: Toward a Corporeal Feminism, Indianapolis : Indiana University Press.

Hamelin, M.1989. Femmes et prison, Montréal : Le Méridien.

Hannah-Moffat, K. et Shaw, M. 2000. An Ideal Prison : Critical Essays on Women's Imprisonment in Canada, Halifax : Fernwood Publishing.

Harding, S. 1986. The Science Question in Feminism, Ithaca : Cornell University Press.

HARDING, S. 1987. Feminism and Methodology, Bloomington : Indiana University Press.

HaRdinc, S. 1991. Whose Science? Whose Knowledge? Ithaca : Cornell University Press.

Heidensohn, F. 1985. Women and Crime, New York : New York University Press.

Heney, J. 1990. Report on Self-Injurious Behaviour in the Kingston Prison for Women, Ottawa : The Correctional Services of Canada.

HORII, G. 1994. "The Art in/of Survival " Journal of Prisoners on Prisons 5 (2) : 6-8.

HORN, D. 1995. "This Norm Which is Not One : Reading the Female Body in Lombroso's Anthropology ", Pp. 109-128 in Deviant Bodies. Critical Perspectives on Difference in Science and Popular Culture, sous la direction de J. Terry, Jennifer et J. Urla. Bloomington : Indiana University Press.

Howe, A. 1994. Punish and Critique: Towards a Feminist Analysis of Penalty, Londres : Routledge.

Hutter, B. et Williams, G. 1981. Controlling Women: The Normal and the Pathological, Londres: Croom Helm.

KeNDALL, K. 1993. Évaluation des services thérapeutiques offerts à la Prison des femmes, Ottawa : Service correctionnel du Canada.

LABADIE, J.-M. 1995. "Corps et crime: De Lavater (1775) à Lombroso (1876) ", Pp.295-345 in Histoire des savoirs sur le crime \& la peine, 1. Des savoirs diffus à la notion de criminel-né, sous la direction de C. Debuyst, F. Digneffe, J.-M. Labadie et A. Pires. Les Presses de l'Université de Montréal, Les Presses de l'Université d'Ottawa, DeBoeck Université.

LAPLANTE, J. 1985. Crime et traitement. Introduction critique à la criminologie, Montréal : Boréal Express.

LAPLANTE, J. 1995. Psychotbérapies \& Impératifs sociaux. Les enjeux de la connaissance de soi, Les Presses de l'Université de Montréal, Les Presses de l'Université d'Ottawa, DeBoeck Université.

LAVIGNE, B. 1999. Corps et enfermement : Récits de femmes, thèse de maîtrise inédite, Département de criminologie, Université d'Ottawa.

Le Breton, D. 2000. Passions du risque, Paris : Éditions Métaillé.

Lombroso, C. et Ferrero, G. 1991 ; lère éd. : 1895. La femme criminelle et la prostituée, Grenoble : Éditions Jérôme Millon. 
MARTEL, J. 1999. Solitude \& Cold Storage: Women's Journeys of Endurance in Segregation, Alberta : Elizabeth Fry Society of Edmonton.

PAILLÉ, P. 1994. "L'analyse par théorisation ancrée " Cabiers de recherche sociologique $23: 147-181$.

Pollack, S. 1993. Opening the Window on a Very Dark Day : A Program Evaluation of the Peer Support Team at the Kingston Prison for Women, thèse de maîtrise inédite, Ottawa, Carleton University.

PRÉJEAN, M. 1994. Sexes et pouvoir : la construction sociale des corps et des émotions, Montréal : Les Presses de l'Université de Montréal.

PROTECTEUR DU CITOYEN. 1985. Le respect des droits des personnes incarcérées, Québec.

Rail, G. 1998. Sport and Postmodern Times, Albany : State University of New York Press.

RamazANOGLU, C. 1993. Up Against Foucault : Explorations of some Tensions Between Foucault and Feminism, Londres : Routledge.

RODRIGUEZ DEL BARRIO, L. 2000. « Le corps et ses mirages : récits et parcours des femmes à travers la folie et sa psychiatrisation ", Pp.165-194 in Du corps des femmes: Contrôle, surveillance et résistance, sous la direction de S. Frigon et M. Kérisit. Ottawa : Les Presses de l'Université d'Ottawa.

Ross, R. et MCKAY, H. 1979. Self-Mutilation, Toronto : Lexington Books.

SCHUR, E. 1984. Labeling Women Deviant: Gender, Stigma, and Social Control, New York : Random House.

SHAw, M. 1991. La détenue au niveau fédéral : Rapport suite à une étude préliminaire [traduction], Ottawa : Ministère du Solliciteur général du Canada, Secteur des affaires correctionnelles.

Service Correctionnel du Canada. 1990. La Création de Choix : Rapport du groupe d'étude sur les femmes purgeant une peine fédérale, Ottawa : Ministète des Approvisionnements et Services.

Service Correctionnel du Canada. 1995. Voix de femmes, Choix de femmes, Rapport du groupe de travail sur la condition des femmes dans le système correctionnel, Ottawa : Ministère des Approvisionnements et Services.

Service Correctionnel du Canada. 1995. Vue d'ensemble : Service Correctionnel du Canada, Établissements fédéraux pour des femmes purgeant une peine fédérale, Ottawa : Service correctionnel du Canada, Programme des femmes purgeant une peine fédérale, Administration centrale.

SMART, C. 1976. Women, Crime and Criminology. A Feminist Critique, London : Routledge and Kegan Paul.

Smith, D. 1987. The Everyday World as Problematic: A Feminist Sociology Boston : Northeastern University Press.

SMITH, D. 1990. “ Femininity as Discourse ", Pp.159-208 in Texts, Facts, and Femininity, Londres: Routledge.

STABLEFORTH, N. 1999. "Des services correctionnels efficaces pour les délinquantes » Forum 11 (3) : 3-5. 
Trevathan, S. 1999. "Les femmes incarcérées dans des établissements fédéraux, provinciaux ou territoriaux $»$ Forum, 11 (3) : 9-12.

VANDELAC, L. 1986. "L'enceinte de la maternité : sexes et sexualités " in Maternité en mouvement : les femmes, la reproduction et les hommes de science, sous la direction de A.M. de Vilaine, L. Gavarini et M. Le Coadic. Montréal : Éditions Saint-Martin.

Welzer-Lang, D., Mathieu, L. et Faure, M. 1997. «Effets de l'incarcération sur le corps et l'estime de soi "Quasimodo : Bulletin trimestriel d'évasion corporelle $2: 21-25$. 